\title{
INEQUALITIES ON GENERALIZED TRIGONOMETRIC FUNCTIONS
}

\author{
Baoju Sun \\ Department of Mathematics
}

Zhejiang University of Water Resources and Electric Power, Hangzhou, Zhejiang 310018, China sunbj@zjweu.edu.cn

Keywords: Generalized trigonometric functions, Cusa-Huygens inequality

Abstract. The Sharp Cusa-Huygens inequality involving the generalized trigonometric functions are established.

\section{Introduction}

It is well known from basic calculus that

$$
\arcsin (x)=\int_{0}^{x} \frac{1}{\left(1-t^{2}\right)^{1 / 2}} \mathrm{~d} t, \quad 0 \leq x \leq 1,
$$

And $\frac{\pi}{2}=\arcsin (1)=\int_{0}^{1} \frac{1}{\left(1-t^{2}\right)^{1 / 2}} \mathrm{~d} t$.

For $1<p<\infty$, We can generalize the above function as follows:

$$
\arcsin _{p}(x) \equiv \int_{0}^{x} \frac{1}{\left(1-t^{p}\right)^{1 / p}} \mathrm{~d} t, \quad 0 \leq x \leq 1,
$$

and $\frac{\pi_{p}}{2}=\arcsin _{p}(1)=\int_{0}^{1} \frac{1}{\left(1-t^{p}\right)^{1 / p}} \mathrm{~d} t$.

where $\pi_{p}=\frac{2 \pi}{p \sin (\pi / p)}$ is decreasing on $(1, \infty)$.

The inverse of $\arcsin _{p}$ on $\left[0, \pi_{p} / 2\right]$ is called the generalized sine function and denoted by $\sin _{p}$. The generalized cosine function $\cos _{p}$ is defined as

$$
\cos _{p}(x) \equiv \frac{\mathrm{d}}{\mathrm{d} x} \sin _{p}(x) .
$$

It is clear from the definition that

$$
\cos _{p}(x)=\left(1-\sin _{p}(x)^{p}\right)^{1 / p} .
$$

The generalized tangent function $\tan _{p}$ is defined as

$$
\tan _{p}(x) \equiv \frac{\sin _{p}(x)}{\cos _{p}(x)} .
$$

It is easy to see that

$$
\frac{\mathrm{d}}{\mathrm{d} x} \cos _{p}(x)=-\cos _{p}(x)^{2-p} \sin _{p}(x)^{p-1}, \frac{\mathrm{d}}{\mathrm{d} x} \tan _{p}(x)=1+\tan _{p}(x)^{p},
$$

when $p=2$, the $p$ - functions $\sin _{p}, \cos _{p}, \tan _{p}$ become our familiar trigonometric functions.

Recently, the generalized trigonometric functions have been studied by many mathematicians from different viewpoints(see [2,4,5,6,7]). In [5,9], the authors gave basic properties of the generalized trigonometric functions. In [6], Klén, Vuorinen and Zhang generalized some classical inequalities for trigonometric functions, such as Mitrinović-Adamović's inequality, Lazarević's inequality, Huygens-type inequalities, and Wilker-type inequalities, to the case of generalized functions. 
The main results of this paper are the following theorems.

Theorem 1 For $1<p \leq 2$, the function

$$
f(x)=\frac{x\left(p+\cos _{p}(x)\right)}{\sin _{p}(x)}
$$

is strictly increasing from $\left(0, \pi_{p} / 2\right)$ onto $\left(p+1, p \pi_{p} / 2\right)$.

Theorem 2 For $1<p \leq 2$, the function

$$
F(x)=\frac{\sin _{p}(x)-x \cos _{p}(x)}{x^{2} \sin _{p}(x)^{p-1} \cos _{p}(x)^{2-p}}
$$

is strictly increasing from $\left(0, \pi_{p} / 2\right)$ onto $\left(\frac{1}{p+1}, b_{p}\right)$.

Where

$$
b_{p}=\left\{\begin{array}{l}
\infty, 1<p<2 \\
\frac{4}{\pi^{2}}, \quad p=2
\end{array}\right.
$$

Theorem 3 For $1<p \leq 2$, the function

$$
G(x)=\frac{\ln \left(\sin _{p}(x) / x\right)}{\ln \left[\left(p+\cos _{p}(x)\right) /(p+1)\right]}
$$

is strictly increasing from $\left(0, \pi_{p} / 2\right)$ onto $\left(1,\left(\log \left(\pi_{p} / 2\right)\right) / \log [(p+1) / p]\right)$.

In particular, for all $p \in(1,2], x \in\left(0, \pi_{p} / 2\right)$,

$$
\left(\frac{p+\cos _{p}(x)}{p+1}\right)^{\alpha}<\frac{\sin _{p} x}{x}<\left(\frac{p+\cos _{p}(x)}{p+1}\right)^{\beta},
$$

Where $a=\left(\log \left(\pi_{p} / 2\right)\right) / \log [(p+1) / p]$ and $\beta=1$ are the best constants.

Remark 4 For $p=2$, the above inequalities are due to C.-P. Chen and W.-S. Cheung [8].

\section{Proof of theorems}

In order to establish our main results we need following lemma:

Lemma 5 ( L'Hopital Monotone Rule see [1] ) Let $-\infty<a<b<\infty$, and let $f, g:[a, b] \rightarrow{ }^{\circ}$ be continuous functions that are differentiable on $(a, b)$, with $f(a)=g(a)=0$ or $f(b)=g(b)=0$. Assume that $g^{\prime}(x) \neq 0$ for each $x \in(a, b)$.

If $f^{\prime} / g^{\prime}$ is increasing (decreasing) on $(a, b)$, then so is $f / g$.

Proof of Theorem1 By differention, we have

$$
f^{\prime}(x)=\frac{1}{\sin _{p}(x)^{2}} g(x)
$$

With $g(x)=p \sin _{p}(x)+\sin _{p}(x) \cos _{p}(x)-p x \cos _{p}(x)-x \cos _{p}(x)^{2-p}$.

a simple computation leads to

$$
\begin{aligned}
& g^{\prime}(x)=\cos _{p}(x)^{2-p}\left[-2 \sin _{p}(x)^{p}+p x \sin _{p}(x)^{p-1}+(2-p) x \cos _{p}(x)^{1-p} \sin _{p}(x)^{p-1}\right] \\
& =\cos _{p}(x)^{2-p} \sin _{p}(x)^{p-1}\left[p x-2 \sin _{p}(x)+(2-p) x \cos _{p}(x)^{1-p}\right]
\end{aligned}
$$




$$
=\cos _{p}(x)^{2-p} \sin _{p}(x)^{p-1} h(x),
$$

where

$$
h(x)=p x-2 \sin _{p}(x)+(2-p) x \cos _{p}(x)^{1-p},
$$

and

$$
\begin{aligned}
& h^{\prime}(x)=p-2 \cos _{p}(x)+(2-p) \cos _{p}(x)^{1-p}+(2-p)(p-1) x \cos _{p}(x)^{2-2 p} \sin _{p}(x)^{p-1} \\
& >(2-p)\left(\cos _{p}(x)^{1-p}-\cos _{p}(x)\right)>0 .
\end{aligned}
$$

Hence $h(x)>h(0)=0$, therefor $g^{\prime}(x)>0, f(x)$ is strictly increasing on $\left(0, \pi_{p} / 2\right)$,

$$
p+1=f\left(0^{+}\right)<f(x)<f\left(\pi_{p} / 2\right)=p \pi_{p} / 2 .
$$

\section{Proof of Theorem 2. Write}

$$
F_{1}(x) \equiv \sin _{p}(x)-x \cos _{p}(x) \text {, and } F_{2}(x) \equiv x^{2} \sin _{p}(x)^{p-1} \cos _{p}(x)^{2-p},
$$

then $F_{1}(0)=0, F_{2}(0)=0$, by simple computations,

$$
\begin{aligned}
& \frac{F_{2}^{\prime}(x)}{F_{1}^{\prime}(x)} \\
= & \frac{2 x \cos _{p}(x)^{2-p} \sin _{p}(x)^{p-1}+(p-1) x^{2} \cos _{p}(x)^{3-p} \sin _{p}(x)^{p-2}+(p-2) x^{2} \cos _{p}(x)^{3-2 p} \sin _{p}(x)^{2 p-2}}{x \cos _{p}(x)^{2-p} \sin _{p}(x)^{p-1}} \\
= & 2+(p-1) x / \tan _{p}(x)+(p-2) x \tan _{p}(x)^{p-1} .
\end{aligned}
$$

Which is strictly decreasing, by lamma $5, \frac{F_{2}(x)}{F_{1}(x)}$ is strictly decreasing on $\left(0, \pi_{p} / 2\right)$,

$F(x)$ is strictly increasing on $\left(0, \pi_{p} / 2\right)$, leads to

$$
\begin{aligned}
& F\left(0^{+}\right)<F(x)<F\left(\pi_{p} / 2\right) \text {. But } \\
& F\left(0^{+}\right)=\lim _{x \rightarrow 0^{+}} \frac{F_{1}(x)}{F_{2}(x)}=\lim _{x \rightarrow 0^{+}} \frac{F_{1}^{\prime}(x)}{F_{2}^{\prime}(x)} \\
& =\lim _{x \rightarrow 0^{+}} \frac{1}{2+(p-1) x / \tan _{p}(x)+(p-2) x \tan _{p}(x)^{p-1}}=\frac{1}{p+1} .
\end{aligned}
$$

Theorem 2 is proved.

Proof of Theorem 3. Write

$$
G_{1}(x) \equiv \ln \frac{\sin _{p} x}{x}, \text { and } G_{2}(x) \equiv \ln \left(\frac{p+\cos _{p}(x)}{p+1}\right),
$$

then $G_{1}(0)=0, G_{2}(0)=0$, by simple computations,

$$
\begin{aligned}
& \frac{G_{1}^{\prime}(x)}{G_{2}^{\prime}(x)}=\frac{\sin _{p}(x)-x \cos _{p}(x)}{x \sin _{p}(x)} \cdot \frac{p+\cos _{p}(x)}{\sin _{p}(x)^{p-1} \cos _{p}(x)^{2-p}} \\
& =\frac{x\left(p+\cos _{p}(x)\right)}{\sin _{p}(x)} \cdot \frac{\sin _{p}(x)-x \cos _{p}(x)}{x^{2} \sin _{p}(x)^{p-1} \cos _{p}(x)^{2-p}}=f(x) F(x) .
\end{aligned}
$$

By theorem 1 and theorem 2, the functions $f(x), F(x)$ are strictly increasing on $\left(0, \pi_{p} / 2\right), f(x) \geq 0, F(x) \geq 0$. Thus

$\frac{G_{1}^{\prime}(x)}{G_{2}^{\prime}(x)}$ is strictly increasing on $\left(0, \pi_{p} / 2\right)$, by lamma 5 , the function

$\frac{G_{1}(x)}{G_{2}(x)}$ is strictly increasing on $\left(0, \pi_{p} / 2\right)$, and we have 


$$
1=G\left(0^{+}\right)<G(x)=\frac{\ln \left(\sin _{p}(x) / x\right)}{\ln \left[\left(p+\cos _{p}(x)\right) /(p+1)\right]}<G\left(\pi_{p} / 2\right)=\frac{\ln \left(\pi_{p} / 2\right)}{\ln ((p+1) / p)} .
$$

Theorem 3 is proved.

\section{References}

[1] G. D. Anderson, M. K. Vamanamurthy, M.Vuorinen, Monotonicity rules in calculus, Amer. Math. Monthly133(2006) 805-816.

[2] G. D. Anderson, M.Vuorinen, X.-H. Zhang , Topics on special functions III. arXiv:1209. 1696v1[math.CA].

[3] E. Neuman, J. Sandor, On Some inequalities involving trigonometric and hyperbolic functions with emphasis on the Cusa-Huygens, Wilker, and Huygens inequalities, Math. Inequal. Appl. 13(2010), 715-423.

[4] B. A. Bhayo, M.Vuorinen, On generalized trigonometric functions with two parameters, J. Approx.Theory 164(2012), 1415-1426.

[5] B. A. Bhayo, M. Vuorinen, Inequalities for eigenfunctions of the p-Laplacian. arXiv:1101.3911v3 [math.CA].

[6] R. Klén, M. Vuorinen, X. -H. Zhang, Inequalities for the generalized trigonometric and hyperbolic functions, J. Math.Anal. and Appl.409 (2014), 521-529.

[7] P. Lindqvist, Note on a nonlinear eigenvalue problem , Rocky Mountain J.Math.23(1993), 281-288.

[8] C. -P. Chen, W. -S. Cheug, Sharp Cusa and Becker-Stark inequalities, J. Inequal. Appl.(2011), Article136(2011).

[9] D. E. Edmunds, P. Gurka, J. Lang. Properties of generalized trigonometric functions, J. Approx. Theory 164(2012), 47-56. 